\title{
Effects of Non-Focused ELF-EMF Treatment on EEG: Preliminary Study
}

\author{
Alessandro Greco, $M D^{*}$ and Alberto Garoli, $M D$ \\ Medical Director, APSP (Public Agency for Personal Health Services) Cles, Italy
}

\begin{abstract}
Introduction: The central nervous system (CNS) is known to be sensitive to focused magnetic stimulation, which is now used routinely for the treatment of various disorders. Extremely Low Frequency Electro Magnetic Fields (ELF-EMF) have been used in various clinical settings and tests however, to date, little is known regarding their functional mechanisms in vivo and, in particular, as a total-body non-focused administration. The CNS acts as the first interactive organ, capable of modulating the effects of ELF-EMF treatment on living systems. This study analysed the effects of 5 different electromagnetic frequency-ranges on the CNS, by studying the endogenous response to exposure. Spectrometry was used to measure the strength of different cerebral wavebands in various brain topographies, together with a component of Z-ratio, as a comparative reference system.
\end{abstract}

Materials and methods: 21 healthy subjects between 20 and 30 years of age were recruited and assessed on two different sessions, one sham day and one treatment day. The subjects were assessed during administration of 5 different ELF-EMF set-ups, using a SEQEX ${ }^{\circledR}$ device, all exposed to the same intensity of $20 \mu \mathrm{T}$ (microtesla), with a protocol duration of 3 minutes, each using a Swipe method, with a progressive increase of $0.1 \mathrm{~Hz}$. The setup range was devised as follows:

- $1-3 \mathrm{~Hz}(\delta)$

- $4-8 \mathrm{~Hz}(\theta)$

- $\quad 9-13 \mathrm{~Hz}(\alpha)$

- $15-29 \mathrm{~Hz}(\beta)$

- $\quad 31-56 \mathrm{~Hz}(\gamma)$

A 14 channel EEG device was used to measure the cortical responses of the 21 subjects during stimulation.

Results: The response to the different electromagnetic stimulation set-ups varied, with activation of different brain derivations observed in EEG. In general, response was greater under stimulation within the $\delta$ range at $1-3 \mathrm{~Hz}$, with a widespread increase in the band strength $\beta(p<0.05)$ and a ubiquitous decrease in the Z-Ratio $(p<0.05)$. Responses were minimal under stimulation in the $\gamma$ range at $31-56 \mathrm{~Hz}$, with a limited reaction in terms of activated areas and band strength, in any case coherent with the $\gamma$ range, particularly in the right hemisphere $(p<0.01)$. Contrary to expectations, no significant increase was observed in the $\alpha$ band.

Conclusions: The electrical activity of the CNS exhibits frequency dependent sensitivity to treatment with ELF-EMF. This observation, if confirmed in further studies and relevant for two main reasons: Firstly, it offers insight for an improved understanding of the mechanisms of action of ELF-EMF on complex biological systems from the perspective of preventing disorders caused by electromagnetic pollution. Secondly it creates several implications for possible integration into existing neurological and psychiatric therapeutic approaches [1].

\section{Introduction}

We dispose today of an ever-increasing evidence of biological interactions with electromagnetic fields and living organisms. Regarding neurological/psychiatric scopes, excellent results have been achieved using Transcranial Magnetic Stimulation (TMS) [2-5]. However, much remains unknown regarding the effects of low frequency, extremely low intensity electromagnetic fields (ELF-EMF) on the cerebral activity of subjects under non-focused, total-body treatment. This ex-
*Corresponding author: Alessandro Greco, Medical Director, APSP (Public Agency for Personal Health Services) Cles, Italy

Accepted: October 12, 2019

Published online: October 14, 2019

Citation: Greco A, Garoli A (2019) Effects of Non-Focused ELFEMF Treatment on EEG: Preliminary Study. Transl Neurosci Res $\operatorname{Rev} 2(1): 38-52$ 
posure simulates the lowest levels of natural environmental exposure of the human body to commonly occurring EMF. The evidence, to date, indicates that local ELF-EMF stimulation modifies cerebral electrical activity, with a significant increase in the alpha waves range, in the frontal regions [5]. Local cranial stimulation with electromagnetic fields in a frequency range of $1-60 \mathrm{~Hz}$ and an intensity scale of 20-100 $\mu \mathrm{T}$, demonstrated the sensitivity of the central nervous system (CNS), expressed as modified cerebral electrical behaviour [69].

The authors decided to observe the effects of non-focused, total body treatment with ELF-EMF on the CNS, in order to clarify:

- Potential environmental exposure to spurious electromagnetic frequencies.

- Potential treatments that might assist diverse medical disciplines.

- Mechanisms of action that lead to measurable behavioural [10] and physiological variations, monitored by Heart Rate Variability (HRV) [11].

It was thus decided to analyse cerebral electrical activity by EEG, as in previous studies listed in the bibliography [6-9]. The aim of the present study was to achieve a qualitative and quantitative understanding regarding non-focused stimulation, identical to the one used to test responses of the autonomic nervous system (ANS) [11], as a specific encephalic electrical reaction.

\section{Materials and Methods}

The study was conducted at the Azienda Pubblica di Servizi alla Persona (APSP) [Public Agency for Personal Health Services] "Santa Maria" retirement home, located in Cles (Trentino, Italy).

For the study, the authors recruited 21 volunteers, healthy subjects between the age of 20 and 30 years: 15 women and 6 men. The subjects were assessed on two different days:

- On one day (sham day) the EEG of the subjects was recorded at rest, with eyes closed, lying on a physiotherapy couch, with a device for generating ELF-EMF switched on but not emitting a field.

- On the other day the EEG of the subjects was recorded at rest, with eyes closed, lying on a physiotherapy couch, with a device for generating ELF-EMF operating and emitting an electromagnetic field.

Throughout the data collection phase, none of the subjects were informed of the nature and aim of the study: The only information provided regarded the type of ELF-EMF therapy to be given (method of administration of treatment, duration) and that EEG measurements that would be taken. None of the volunteers on either of the days were informed whether or not there was an actual administration of ELFEMF. All the measurements were taken between 3 p.m. and 6 p.m.
A SEQEX ${ }^{\circledR}$ device was used for the study, produced and distributed by the Italian company S.I.S.T.E.M.I. Srl. (Trento, Italy), certified CSQ ISO-13485. These devices produce complex electromagnetic fields, using an analogue mechanism in a range of frequencies from 1 to $80 \mathrm{~Hz}$, and variable intensities from 1 to $20 \mu \mathrm{T}$. The field parameters were tested by the manufacturing company using specialized equipment: A GM08 Gaussmeter produced by the Hirst company. The electromagnetic field produced by the device control unit (on which the parameters of the electromagnetic field are set), is emitted from a mat containing a Helmholtz coil that generates the ELF-EMF. Individual patients were asked to lie on the mat to receive non-focused total-body treatment with weak electromagnetic fields.

This study tested 5 different electromagnetic set-ups, each of which included 3 frequency/intensity pairs named "steps", with a time-on administration and a pause or timeoff cycle (expressed in seconds). A sinusoidal wave form was used to produce the emissions for all the steps. The 5 different set-ups were labelled with Greek letters corresponding to the brain waves of the same frequencies $(\delta, \theta, \alpha, \beta, \gamma)$. The characteristics of the individual set-ups are listed in the following Table 1.

All the set-ups had a treatment time of 3 minutes. The subjects were assessed without administration of any ELFEMF, but with the device switched on (measurement labelled $\omega$ ), and then subsequently a single time for each set-up listed in the Table 1 . Each subject involved in the study was therefore assessed 6 times.

The volunteers were assessed lying down with eyes closed. The position of the wooden couch supporting the mat was arranged so that the subjects were unable to see the control unit. This expedient, combined with the absence of any perception when the field is generated, ensured that the subjects did not know whether or not the device was actually in operation. After each distinct assessment with the electromagnetic field, the subjects were asked to stand up and remain upright for 3 minutes, away from the device mat. All administrations were conducted using the same device and in the same environment, the integrated medicine clinic at the APSP Santa Maria (Cles), without distractions or stimulations from third parties (background music, air fresheners, etc.) or the medical staff.

\section{EEG}

Electroencephalography is the most widely used non-invasive method for the study of cerebral electrical activity [12]. An electroencephalogram trace (EEG) divides up cerebral electrical activity into different brain wave ranges [12] labelled with the Greek letters: $\delta, \theta, \alpha, \beta, \gamma$.

In this study an Eopch+ $[13,14] 14$ channel wireless electroencephalography headset from the Emotiv ${ }^{\circledR}$ company, was used. Sample recording was set at $128 \mathrm{~Hz}$. The following brain derivations were studied: AF3, F7, F3, FC5, T7, P7, O1, O2, P8, T8, FC6, F4, F8, AF4. 


\section{EEG: FFT and Power Band}

The device operates with the EmotivPRO proprietary software, which is capable of recording a RAW trace and conducting real-time analysis of band strength by Fourier transform (FFT). In this way it is possible to measure the band strength for each cerebral wave except delta waves, which are not analysed by FFT because the strength of delta signal would possibly mask high frequency low amplitude oscillations. The Power Band reading was assessed for all the brain derivations studied. The emissions of the two individual hemispheres as the sum of same-side derivations, and overall brain emissions, were also assessed as more generic readings.

The software did not provide data for the endogenous response to the $\delta$ wave, while it subdivided the study of $\beta$ waves into two subcategories: low and high. Consequently, the bands recorded were:

- $\theta(4-8 \mathrm{~Hz})$,

- $\alpha(8-12 \mathrm{~Hz})$,

- $\beta$-low (12-16 Hz),

- $\beta$-high (16-25 Hz),

- $\gamma(25-45 \mathrm{~Hz})$.

\section{EEG: Z-Ratio}

In order to resolve the information gap regarding any incidental modifications to the $\delta$ waves, the data obtained by the abovementioned software were downloaded in ${ }^{*}$.EDF format and analysed using the open source EDFBrowser software. This was used to analyse the Z-Ratio [15], taken here as a measurement to calculate cerebral activity at low and high frequencies within a period or "epoch" of 2 seconds. This period is expressed on a scale of -1 to +1 , with negative values indicating a predominance of high frequency activity and positive values indicating a predominance of low frequency activity. The Z-Ratio is calculated as follows (the Greek letters represent the corresponding brain waves):

$$
\text { Z-ratio }=\frac{(\delta+\theta)-(\alpha+\beta)}{\delta+\theta+\alpha+\beta}
$$

The data obtained for the Power Bands did not follow a Gaussian distribution and were consequently transformed using the Log in order to normalize the distribution and make it possible to conduct a parametric statistical analysis applying Student's t-distribution. The Z-ratio data again did not have a Gaussian distribution and they were analysed using the Mann-Whitney test. Transformation to a logarithmic scale and statistical analysis were conducted using the Graph$\mathrm{Pad}^{\circledR}$ InStat software.

\section{Results}

Shown below are the average power band values for the cerebral waves $(\theta, \alpha, \beta$-low, $\beta$-high, $\gamma)$ for each derivation (Table 2, Table 3, Table 4, Table 5 and Table 6) (Graph 1, Graph 2, Graph 3, Graph 4 and Graph 5), and the Z-Ratio (Table 7 and Graph 6) obtained from the 6 measurements.

\section{Effects of stimulation with ELF-EMF $\delta$}

Stimulation with frequencies in the $\delta$ range $(1-2-3 \mathrm{~Hz})$ produced an interesting increase in cerebral electrical activity in general, with an adaptive response. In detail:

- AF3: Decrease in Z-Ratio (average value $\omega: 0.055$; average value under ELF-EMF: -0.12, $\mathrm{p}=0.003$ );

- F7: Decrease in $\theta$ wave (average value $\omega: 5.117$; average value under ELF-EMF: 2.168, $p=0.039$ ), decrease in Z-Ratio (average value $\omega: 0.063$; average value under ELF-EMF: $-0.102, p=0.0023)$;

- F3: Increase in $\beta$-low wave (average value $\omega$ : 0.476; av-

Table 1: The table lists the electromagnetic fields administered. Each step lasted for 1 minute.

\begin{tabular}{|c|c|c|c|c|c|}
\hline Set-Up & Step & Intensity $(\mu T)$ & Frequency $(\mathrm{Hz})$ & Time-On (s) & Time-Off (s) \\
\hline \multirow[t]{3}{*}{$\delta$} & 1 & 20 & 1 & 5 & 1 \\
\hline & 2 & 20 & 2 & 5 & 1 \\
\hline & 3 & 20 & 3 & 5 & 1 \\
\hline \multirow[t]{3}{*}{$\theta$} & 1 & 20 & 4 & 5 & 1 \\
\hline & 2 & 20 & 6 & 5 & 1 \\
\hline & 3 & 20 & 8 & 5 & 1 \\
\hline \multirow[t]{3}{*}{$\alpha$} & 1 & 20 & 9 & 5 & 1 \\
\hline & 2 & 20 & 11 & 5 & 1 \\
\hline & 3 & 20 & 13 & 5 & 1 \\
\hline \multirow[t]{3}{*}{$\beta$} & 1 & 20 & 15 & 5 & 1 \\
\hline & 2 & 20 & 22 & 5 & 1 \\
\hline & 3 & 20 & 29 & 5 & 1 \\
\hline \multirow[t]{3}{*}{$v$} & 1 & 20 & 31 & 5 & 1 \\
\hline & 2 & 20 & 43 & 5 & 1 \\
\hline & 3 & 20 & 56 & 5 & 1 \\
\hline
\end{tabular}


Table 2: Table of average values obtained from the 6 different measurements of the $\theta$ brain wave power band (stimulated by ELF-EMF except for $\omega$ ). Each column shows a different endogenous electromagnetic response, with the exception of $\omega$.

\begin{tabular}{|c|c|c|c|c|c|c|}
\hline Band $\theta$ & $\omega$ & $\delta$ & $\theta$ & $\alpha$ & $\beta$ & $\boldsymbol{v}$ \\
\hline AF3 & $3.60 \pm 4.22$ & $2.77 \pm 3.55$ & $2.77 \pm 3.65$ & $1.69 \pm 1.82$ & $3.25 \pm 1.56$ & $3.27 \pm 1.43$ \\
\hline F7 & $5.12 \pm 2.98$ & $2.17 \pm 1.44$ & $3.08 \pm 1.79$ & $2.52 \pm 2.56$ & $6.12 \pm 8.79$ & $4.06 \pm 3.13$ \\
\hline F3 & $1.36 \pm 2.02$ & $0.98 \pm 0.40$ & $1.27 \pm 1.63$ & $1.58 \pm 2.23$ & $3.43 \pm 2.63$ & $3.51 \pm 3.34$ \\
\hline FC5 & $1.72 \pm 1.45$ & $1.02 \pm 0.77$ & $0.99 \pm 1.29$ & $0.69 \pm 0.53$ & $0.88 \pm 0.80$ & $0.79 \pm 0.57$ \\
\hline T7 & $4.46 \pm 17.13$ & $1.70 \pm 1.46$ & $1.28 \pm 1.79$ & $2.43 \pm 3.68$ & $2.57 \pm 7.08$ & $1.08 \pm 1.90$ \\
\hline P7 & $1 \pm 1.25$ & $2.78 \pm 12.84$ & $0.71 \pm 0.60$ & $0.82 \pm 0.68$ & $0.87 \pm 0.57$ & $1.05 \pm 2.14$ \\
\hline 01 & $0.87 \pm 0.88$ & $2.84 \pm 9.91$ & $1.58 \pm 1.14$ & $1.79 \pm 4.49$ & $1.37 \pm 0.67$ & $1.06 \pm 0.87$ \\
\hline 02 & $1.27 \pm 0.97$ & $1.01 \pm 1.21$ & $1.58 \pm 1.57$ & $1.20 \pm 1.41$ & $0.93 \pm 0.77$ & $1.26 \pm 1.61$ \\
\hline P8 & $1.06 \pm 0.92$ & $0.89 \pm 0.63$ & $1.99 \pm 1.82$ & $4.07 \pm 12.96$ & $1.07 \pm 0.32$ & $2.14 \pm 4.60$ \\
\hline T8 & $2.29 \pm 2.50$ & $1.65 \pm 1.92$ & $2.29 \pm 1.43$ & $1.72 \pm 1.83$ & $2.37 \pm 3.07$ & $1.90 \pm 1.32$ \\
\hline FC6 & $2.49 \pm 4.17$ & $1.63 \pm 1.98$ & $1.81 \pm 1.36$ & $1.07 \pm 1.14$ & $1.52 \pm 1.34$ & $1.71 \pm 2.24$ \\
\hline F4 & $1.76 \pm 3.25$ & $1.42 \pm 1.12$ & $1.88 \pm 2.90$ & $1.08 \pm 1.13$ & $1.52 \pm 1.05$ & $1.67 \pm 1.32$ \\
\hline F8 & $5.37 \pm 12.64$ & $3.58 \pm 5.34$ & $2.80 \pm 2.44$ & $3.16 \pm 3.74$ & $4.56 \pm 3.77$ & $4.69 \pm 1.20$ \\
\hline AF4 & $8.59 \pm 24.76$ & $2.67 \pm 4.62$ & $4.63 \pm 6.57$ & $3.16 \pm 2.52$ & $5.15 \pm 3.15$ & $5.24 \pm 3.09$ \\
\hline LH Hemisphere & $2.51 \pm 2.23$ & $1.88 \pm 1.96$ & $1.60 \pm 0.85$ & $1.49 \pm 1.02$ & $2.59 \pm 1.44$ & $2.03 \pm 0.85$ \\
\hline RH Hemisphere & $3.48 \pm 6.54$ & $1.91 \pm 1.91$ & $2.51 \pm 1.63$ & $2.34 \pm 1.99$ & $2.54 \pm 1.37$ & $2.84 \pm 1.66$ \\
\hline Total Brain & $3.13 \pm 3.91$ & $2.02 \pm 1.53$ & $2.14 \pm 1.12$ & $2.04 \pm 1.30$ & $2.69 \pm 1.36$ & $2.54 \pm 1.12$ \\
\hline
\end{tabular}

Table 3: Table of average values obtained from the 6 different measurements of the $\alpha$ brain wave power band (stimulated by ELF-EMF with the exception of $\omega$ ). Each column shows a different endogenous electromagnetic response, with the exception of $\omega$.

\begin{tabular}{|c|c|c|c|c|c|c|}
\hline$\alpha$ band & $\omega$ & $\delta$ & $\theta$ & $\alpha$ & $\boldsymbol{\beta}$ & $v$ \\
\hline AF3 & $2.27 \pm 2.65$ & $2.18 \pm 2.38$ & $2.48 \pm 1.23$ & $1.37 \pm 0.99$ & $1.74 \pm 2.63$ & $1.48 \pm 0.57$ \\
\hline F7 & $1.7 \pm 1.19$ & $1.48 \pm 0.58$ & $3.31 \pm 2.26$ & $1.58 \pm 0.89$ & $2.65 \pm 2.58$ & $1.44 \pm 0.83$ \\
\hline F3 & $1.56 \pm 1.06$ & $0.97 \pm 0.54$ & $1.56 \pm 2.70$ & $1.49 \pm 2.43$ & $2.01 \pm 4.23$ & $0.91 \pm 0.32$ \\
\hline FC5 & $1.37 \pm 1.15$ & $0.82 \pm 0.66$ & $1.33 \pm 1.81$ & $0.65 \pm 0.44$ & $0.82 \pm 0.95$ & $0.49 \pm 0.28$ \\
\hline $\mathrm{T7}$ & $3.63 \pm 16.53$ & $1.26 \pm 1.43$ & $1.42 \pm 1.54$ & $1.52 \pm 2.28$ & $1.82 \pm 5.09$ & $0.70 \pm 0.86$ \\
\hline P7 & $0.77 \pm 0.78$ & $3.09 \pm 14.23$ & $1.17 \pm 0.76$ & $0.79 \pm 0.67$ & $1.04 \pm 1.10$ & $0.79 \pm 0.70$ \\
\hline 01 & $1.99 \pm 1.07$ & $1.97 \pm 1.32$ & $2.89 \pm 2.67$ & $1.28 \pm 1.65$ & $1.48 \pm 1.94$ & $1.23 \pm 1.11$ \\
\hline 02 & $1.89 \pm 1.36$ & $4.81 \pm 9.48$ & $3.87 \pm 2.48$ & $1.98 \pm 1.78$ & $3.08 \pm 7.77$ & $2.23 \pm 1.40$ \\
\hline P8 & $1.32 \pm 0.86$ & $2.43 \pm 4.48$ & $3.68 \pm 2.27$ & $3.93 \pm 4.64$ & $1.71 \pm 0.99$ & $2.73 \pm 4.52$ \\
\hline T8 & $1.95 \pm 2.00$ & $2.77 \pm 3.50$ & $3.61 \pm 1.76$ & $1.68 \pm 1.19$ & $2.05 \pm 1.43$ & $1.58 \pm 0.85$ \\
\hline FC6 & $1.53 \pm 1.90$ & $1.84 \pm 2.94$ & $2.38 \pm 1.83$ & $1.21 \pm 0.76$ & $1.31 \pm 1.59$ & $1.29 \pm 0.93$ \\
\hline F4 & $0.98 \pm 0.73$ & $1.37 \pm 1.03$ & $1.76 \pm 2.33$ & $1.43 \pm 1.56$ & $1.19 \pm 2.51$ & $1.27 \pm 1.20$ \\
\hline F8 & $2.39 \pm 3.04$ & $2.76 \pm 3.81$ & $3.46 \pm 3.17$ & $2.23 \pm 2.03$ & $1.78 \pm 4.50$ & $1.62 \pm 0.49$ \\
\hline AF4 & $3.26 \pm 4.35$ & $2.29 \pm 2.85$ & $4.83 \pm 4.93$ & $2.09 \pm 3.35$ & $1.75 \pm 1.99$ & $1.12 \pm 0.55$ \\
\hline LH Hemisphere & $1.60 \pm 2.08$ & $1.57 \pm 1.94$ & $1.81 \pm 1.18$ & $1.08 \pm 0.74$ & $1.6 \pm 1.47$ & $0.89 \pm 0.33$ \\
\hline RH Hemisphere & $1.92 \pm 1.67$ & $2.72 \pm 2.71$ & $3.12 \pm 1.41$ & $2.15 \pm 1.21$ & $1.92 \pm 1.56$ & $1.67 \pm 0.88$ \\
\hline Total Brain & $1.85 \pm 1.79$ & $2.23 \pm 2.12$ & $2.55 \pm 1.29$ & $1.69 \pm 0.88$ & $1.83 \pm 1.55$ & $1.33 \pm 0.59$ \\
\hline
\end{tabular}

erage value under ELF-EMF: 1.058, $p=0.04)$, increase in $\beta$-high wave (average value $\omega$ : 0.338; average value under ELF-EMF: $1.195, p=0.047$ ), increase in $\gamma$ wave (average value $\omega$ : 0.229 ; average value under ELF-EMF: $0.632, p=$ 0.028 ), decrease in Z-Ratio (average value $\omega: 0.038$; average value under ELF-EMF: $-0.23, p=0.000$ );
- FC5: Decrease in Z-Ratio (average value $\omega: 0.087$; average value under ELF-EMF: -0.146, $\mathrm{p}=0.000$ );

- T7: Decrease in Z-Ratio (average value $\omega: 0.013$; average value under ELF-EMF: $-0.146, p=0.016)$;

- P7: Increase in $\gamma$ wave (average value $\omega$ : 0.152; average 
Table 4: Table of average values obtained from the 6 different measurements of the $\beta$-low brain wave power band (stimulated by ELF-EMF with the exception of $\omega$ ). Each column shows a different endogenous electromagnetic response, with the exception of $\omega$.

\begin{tabular}{|c|c|c|c|c|c|c|}
\hline$\beta$-low band & $\omega$ & $\delta$ & $\theta$ & $\alpha$ & $\beta$ & $v$ \\
\hline AF3 & $0.74 \pm 0.79$ & $1.50 \pm 1.89$ & $1.47 \pm 0.52$ & $0.74 \pm 0.44$ & $1.16 \pm 1.63$ & $0.66 \pm 0.45$ \\
\hline F7 & $0.78 \pm 0.53$ & $1.37 \pm 0.25$ & $2.06 \pm 1.14$ & $1.18 \pm 1.05$ & $1.75 \pm 1.66$ & $1.11 \pm 1.06$ \\
\hline F3 & $0.48 \pm 0.36$ & $1.06 \pm 1.05$ & $0.93 \pm 1.58$ & $0.99 \pm 1.21$ & $1.35 \pm 2.30$ & $0.51 \pm 0.58$ \\
\hline FC5 & $0.45 \pm 0.35$ & $0.67 \pm 0.63$ & $0.57 \pm 1.03$ & $0.63 \pm 1.28$ & $0.53 \pm 0.25$ & $0.28 \pm 0.17$ \\
\hline $\mathrm{T7}$ & $1.34 \pm 4.22$ & $1.35 \pm 0.66$ & $0.7 \pm 0.87$ & $1.23 \pm 1.27$ & $0.9 \pm 0.49$ & $0.52 \pm 0.34$ \\
\hline P7 & $0.38 \pm 0.41$ & $3.07 \pm 14.3$ & $0.43 \pm 0.39$ & $2.70 \pm 12.17$ & $0.47 \pm 0.54$ & $0.49 \pm 0.52$ \\
\hline 01 & $0.87 \pm 0.47$ & $2.75 \pm 4.08$ & $1.93 \pm 2.18$ & $0.95 \pm 0.59$ & $2.03 \pm 1.77$ & $1.07 \pm 1.54$ \\
\hline 02 & $0.77 \pm 0.38$ & $3.12 \pm 7.02$ & $2.29 \pm 3.79$ & $1.17 \pm 0.76$ & $1.68 \pm 2.46$ & $1.31 \pm 1.41$ \\
\hline P8 & $0.97 \pm 0.48$ & $2.63 \pm 0.47$ & $2.02 \pm 1.14$ & $1.62 \pm 1.52$ & $1.57 \pm 0.48$ & $1.43 \pm 3.43$ \\
\hline T8 & $0.72 \pm 0.38$ & $2.48 \pm 1.57$ & $2.73 \pm 1.69$ & $1.44 \pm 1.03$ & $1.79 \pm 1.79$ & $1.01 \pm 0.73$ \\
\hline FC6 & $0.78 \pm 0.74$ & $1.51 \pm 1.77$ & $1.19 \pm 0.37$ & $0.67 \pm 0.88$ & $0.81 \pm 0.50$ & $0.75 \pm 0.60$ \\
\hline F4 & $0.79 \pm 2.29$ & $0.76 \pm 0.47$ & $1.37 \pm 1.25$ & $0.77 \pm 1.38$ & $0.79 \pm 0.37$ & $0.75 \pm 0.62$ \\
\hline F8 & $0.90 \pm 0.62$ & $3.16 \pm 2.88$ & $1.17 \pm 0.86$ & $1.88 \pm 1.10$ & $1.09 \pm 0.80$ & $1.01 \pm 0.91$ \\
\hline AF4 & $0.89 \pm 1.07$ & $2.87 \pm 2.97$ & $2.73 \pm 1.92$ & $1.93 \pm 0.77$ & $1.49 \pm 0.72$ & $1.18 \pm 1.62$ \\
\hline LH Hemisphere & $0.6 \pm 0.55$ & $1.58 \pm 2.11$ & $1.07 \pm 0.85$ & $1.13 \pm 1.68$ & $1.09 \pm 0.63$ & $0.59 \pm 0.48$ \\
\hline RH Hemisphere & $0.79 \pm 0.59$ & $2.51 \pm 1.64$ & $1.70 \pm 0.85$ & $1.39 \pm 0.60$ & $1.35 \pm 0.55$ & $1.04 \pm 0.93$ \\
\hline Total Brain & $0.73 \pm 0.51$ & $2.14 \pm 1.86$ & $1.44 \pm 0.83$ & $1.34 \pm 1.07$ & $1.28 \pm 0.60$ & $0.85 \pm 0.64$ \\
\hline
\end{tabular}

Table 5: Table of average values obtained from the 6 different measurements of the $\beta$-high brain wave power band (stimulated by ELF-EMF with the exception of $\omega$ ). Each column shows a different endogenous electromagnetic response, with the exception of $\omega$.

\begin{tabular}{|c|c|c|c|c|c|c|}
\hline Band $\beta$-high & $\omega$ & $\delta$ & $\theta$ & $\alpha$ & $\boldsymbol{\beta}$ & $v$ \\
\hline AF3 & $0.53 \pm 0.79$ & $0.82 \pm 0.61$ & $0.97 \pm 0.42$ & $1.26 \pm 0.81$ & $0.75 \pm 0.45$ & $0.53 \pm 0.66$ \\
\hline F7 & $0.43 \pm 0.46$ & $1.31 \pm 0.33$ & $2.03 \pm 1.33$ & $1.78 \pm 0.56$ & $1.22 \pm 0.99$ & $0.75 \pm 1.17$ \\
\hline F3 & $0.34 \pm 0.16$ & $1.19 \pm 1.56$ & $1.33 \pm 2.17$ & $1.61 \pm 1.36$ & $0.84 \pm 0.60$ & $0.49 \pm 0.28$ \\
\hline FC5 & $0.33 \pm 0.35$ & $0.51 \pm 0.35$ & $0.56 \pm 1.52$ & $0.51 \pm 0.89$ & $0.48 \pm 0.37$ & $0.27 \pm 0.21$ \\
\hline T7 & $1.08 \pm 2.09$ & $1.14 \pm 0.71$ & $1.49 \pm 0.59$ & $1.89 \pm 0.63$ & $1.16 \pm 1.17$ & $0.42 \pm 0.37$ \\
\hline P7 & $0.31 \pm 0.28$ & $2.37 \pm 11.16$ & $0.59 \pm 0.39$ & $0.68 \pm 0.54$ & $0.43 \pm 0.46$ & $0.38 \pm 0.53$ \\
\hline 01 & $0.49 \pm 0.37$ & $1.23 \pm 0.28$ & $1.19 \pm 1.66$ & $1.66 \pm 1.02$ & $0.91 \pm 0.42$ & $0.46 \pm 0.51$ \\
\hline 02 & $0.33 \pm 0.15$ & $1.05 \pm 0.79$ & $0.71 \pm 0.79$ & $0.64 \pm 0.68$ & $0.78 \pm 0.34$ & $0.62 \pm 0.82$ \\
\hline P8 & $0.39 \pm 0.50$ & $1.72 \pm 0.4$ & $1.61 \pm 0.79$ & $1.88 \pm 0.31$ & $0.68 \pm 0.28$ & $0.49 \pm 0.49$ \\
\hline T8 & $1.11 \pm 0.42$ & $1.99 \pm 0.86$ & $1.78 \pm 1.68$ & $2.03 \pm 0.46$ & $1.33 \pm 2.08$ & $0.82 \pm 1.37$ \\
\hline FC6 & $0.66 \pm 0.82$ & $0.81 \pm 0.49$ & $0.63 \pm 0.43$ & $0.60 \pm 0.42$ & $0.67 \pm 0.25$ & $0.41 \pm 0.48$ \\
\hline F4 & $0.47 \pm 1.03$ & $0.78 \pm 0.57$ & $1.33 \pm 1.17$ & $1.07 \pm 0.29$ & $0.52 \pm 0.34$ & $0.56 \pm 0.39$ \\
\hline F8 & $0.75 \pm 0.58$ & $2.37 \pm 1.13$ & $2.19 \pm 0.94$ & $2.04 \pm 0.91$ & $0.79 \pm 0.66$ & $0.68 \pm 1.13$ \\
\hline AF4 & $0.78 \pm 1.69$ & $2.35 \pm 0.59$ & $2.55 \pm 1.59$ & $2.50 \pm 1.39$ & $1.22 \pm 1.63$ & $0.82 \pm 0.95$ \\
\hline LH Hemisphere & $0.47 \pm 0.33$ & $1.18 \pm 1.43$ & $1.15 \pm 0.91$ & $1.31 \pm 0.65$ & $0.79 \pm 0.39$ & $0.44 \pm 0.39$ \\
\hline RH Hemisphere & $0.67 \pm 0.58$ & $1.69 \pm 0.42$ & $1.64 \pm 0.76$ & $1.59 \pm 0.46$ & $0.89 \pm 0.52$ & $0.64 \pm 0.59$ \\
\hline Total Brain & $0.59 \pm 0.42$ & $1.50 \pm 0.89$ & $1.45 \pm 0.86$ & $1.52 \pm 0.57$ & $0.88 \pm 0.46$ & $0.56 \pm 0.51$ \\
\hline
\end{tabular}

value under ELF-EMF: $0.867, \mathrm{p}=0.017)$, decrease in Z-Ratio (average value $\omega: 0.04$; average value under ELF-EMF: $-0.159, p=0.000)$;

- 01: Decrease in Z-Ratio (average value $\omega: 0.039$; average value under ELF-EMF: $-0.148, p=0.007)$;

- 02: Increase in $\beta$-low wave (average value $\omega$ : 0.765 ; av- erage value under ELF-EMF: 3.312, $p=0.05)$, increase in $\beta$-high wave (average value $\omega: 0.334$; average value under ELF-EMF: $1.053, p=0.009$ ), increase in $\gamma$ wave (average value $\omega$ : 0.132 ; average value under ELF-EMF: $0.318, p=$ 0.012 ), decrease in Z-Ratio (average value $\omega:-0.007$; average value under ELF-EMF: $-0.18, p=0.002$ ); 
Table 6: Table of average values obtained from the 6 different measurements of the $\gamma$ brain wave power band (stimulated by ELF-EMF with the exception of $\omega$ ). Each column shows a different endogenous electromagnetic response, with the exception of $\omega$.

\begin{tabular}{|c|c|c|c|c|c|c|}
\hline Band $y$ & $\omega$ & $\delta$ & $\theta$ & $\alpha$ & $\beta$ & 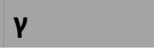 \\
\hline AF3 & $0.59 \pm 1.12$ & $0.51 \pm 0.55$ & $0.84 \pm 0.35$ & $0.59 \pm 1.00$ & $1.03 \pm 1.52$ & $0.29 \pm 0.17$ \\
\hline F7 & $0.27 \pm 0.17$ & $0.56 \pm 0.30$ & $1.24 \pm 0.79$ & $0.67 \pm 0.51$ & $1.04 \pm 0.58$ & $0.54 \pm 1.00$ \\
\hline F3 & $0.23 \pm 0.11$ & $0.63 \pm 0.51$ & $0.7 \pm 0.45$ & $0.97 \pm 1.63$ & $0.89 \pm 0.33$ & $0.31 \pm 0.31$ \\
\hline FC5 & $0.25 \pm 0.13$ & $0.24 \pm 0.27$ & $0.24 \pm 0.29$ & $0.38 \pm 0.81$ & $0.57 \pm 0.44$ & $0.29 \pm 0.33$ \\
\hline $\mathrm{T7}$ & $0.49 \pm 0.96$ & $0.47 \pm 0.26$ & $0.16 \pm 1.23$ & $0.73 \pm 0.87$ & $1.46 \pm 2.24$ & $0.32 \pm 0.21$ \\
\hline P7 & $0.15 \pm 0.12$ & $0.87 \pm 3.11$ & $0.93 \pm 0.25$ & $0.51 \pm 0.66$ & $0.48 \pm 0.23$ & $0.83 \pm 2.65$ \\
\hline 01 & $0.39 \pm 0.15$ & $0.64 \pm 0.31$ & $0.56 \pm 0.62$ & $0.55 \pm 0.68$ & $1.20 \pm 0.86$ & $0.39 \pm 0.61$ \\
\hline 02 & $0.13 \pm 0.07$ & $0.32 \pm 0.23$ & $0.52 \pm 0.33$ & $0.28 \pm 0.28$ & $0.63 \pm 0.29$ & $0.36 \pm 0.70$ \\
\hline P8 & $0.35 \pm 0.27$ & $0.67 \pm 0.19$ & $1.66 \pm 0.66$ & $0.44 \pm 0.22$ & $1.21 \pm 0.09$ & $0.39 \pm 0.59$ \\
\hline T8 & $0.49 \pm 0.30$ & $0.87 \pm 0.33$ & $1.34 \pm 0.73$ & $0.6 \pm 0.42$ & $0.86 \pm 0.42$ & $0.68 \pm 1.63$ \\
\hline FC6 & $0.42 \pm 0.88$ & $0.57 \pm 0.16$ & $0.56 \pm 0.19$ & $0.36 \pm 0.42$ & $0.81 \pm 0.10$ & $0.31 \pm 0.35$ \\
\hline F4 & $0.15 \pm 0.16$ & $0.45 \pm 0.29$ & $1.12 \pm 2.13$ & $0.43 \pm 0.36$ & $0.45 \pm 0.35$ & $0.51 \pm 0.51$ \\
\hline F8 & $0.41 \pm 0.51$ & $1.12 \pm 0.58$ & $1.92 \pm 0.37$ & $0.86 \pm 1.02$ & $1.15 \pm 0.25$ & $0.47 \pm 0.56$ \\
\hline AF4 & $0.72 \pm 0.78$ & $1.26 \pm 0.81$ & $2.28 \pm 3.19$ & $1.16 \pm 1.38$ & $1.42 \pm 0.98$ & $0.77 \pm 1.12$ \\
\hline LH Hemisphere & $0.33 \pm 0.21$ & $0.53 \pm 0.45$ & $0.76 \pm 0.44$ & $0.59 \pm 0.63$ & $0.91 \pm 0.54$ & $0.41 \pm 0.58$ \\
\hline RH Hemisphere & $0.41 \pm 0.66$ & $0.79 \pm 0.42$ & $1.48 \pm 0.88$ & $0.63 \pm 0.42$ & $0.99 \pm 0.25$ & $0.51 \pm 0.63$ \\
\hline Total Brain & $0.38 \pm 0.43$ & $0.69 \pm 0.38$ & $1.16 \pm 0.56$ & $0.65 \pm 0.51$ & $0.99 \pm 0.40$ & $0.48 \pm 0.64$ \\
\hline
\end{tabular}

Table 7: Table of average values obtained from the 6 different measurements of the Z-ratio (stimulated by ELF-EMF with the exception of $\omega$ ). Each column shows a different endogenous electromagnetic response, with the exception of $\omega$.

\begin{tabular}{|c|c|c|c|c|c|c|}
\hline Z-ratio & $\omega$ & $\delta$ & $\theta$ & $\alpha$ & $\beta$ & $\gamma$ \\
\hline AF3 & $0.06 \pm 0.07$ & $-0.12 \pm 0.30$ & $0.08 \pm 0.24$ & $-0.01 \pm 0.19$ & $0.08 \pm 0.16$ & $-0.05 \pm 0.29$ \\
\hline F7 & $0.06 \pm 0.04$ & $-0.10 \pm 0.22$ & $0.11 \pm 0.23$ & $0.08 \pm 0.23$ & $0.11 \pm 0.26$ & $0.03 \pm 0.25$ \\
\hline F3 & $0.04 \pm 0.19$ & $-0.23 \pm 0.25$ & $0.14 \pm 0.2$ & $-0.11 \pm 0.29$ & $0.26 \pm 0.09$ & $-0.01 \pm 0.25$ \\
\hline FC5 & $0.09 \pm 0.09$ & $-0.15 \pm 0.26$ & $0.11 \pm 0.20$ & $0.04 \pm 0.25$ & $0.01 \pm 0.26$ & $0.03 \pm 0.26$ \\
\hline $\mathrm{T7}$ & $0.013 \pm 0.20$ & $-0.15 \pm 0.27$ & $0.15 \pm 0.22$ & $0.12 \pm 0.22$ & $0.01 \pm 0.13$ & $0.04 \pm 0.25$ \\
\hline P7 & $0.04 \pm 0.16$ & $-0.16 \pm 0.22$ & $0.18 \pm 0.23$ & $-0.04 \pm 0.23$ & $-0.02 \pm 0.17$ & $0.02 \pm 0.29$ \\
\hline 01 & $0.04 \pm 0.25$ & $-0.15 \pm 0.21$ & $0.17 \pm 0.28$ & $-0.04 \pm 0.27$ & $0.05 \pm 0.14$ & $0.03 \pm 0.31$ \\
\hline 02 & $-0.01 \pm 0.11$ & $-0.18 \pm 0.25$ & $0.19 \pm 0.30$ & $-0.06 \pm 0.17$ & $0.05 \pm 0.19$ & $0.05 \pm 031$ \\
\hline P8 & $0.019 \pm 0.20$ & $-0.16 \pm 0.19$ & $0.16 \pm 0.22$ & $-0.05 \pm 0.28$ & $0.03 \pm 0.26$ & $-0.03 \pm 0.21$ \\
\hline T8 & $0.10 \pm 0.24$ & $-0.19 \pm 0.35$ & $0.21 \pm 0.19$ & $-0.05 \pm 0.19$ & $0.03 \pm 0.14$ & $-0.02 \pm 0.20$ \\
\hline FC6 & $0.09 \pm 0.08$ & $-0.27 \pm 0.34$ & $0.13 \pm 0.16$ & $0.05 \pm 0.12$ & $0.07 \pm 0.28$ & $-0.02 \pm 0.22$ \\
\hline F4 & $0.16 \pm 0.26$ & $-0.28 \pm 0.28$ & $0.21 \pm 0.28$ & $-0.12 \pm 0.22$ & $-0.02 \pm 0.16$ & $-0.01 \pm 0.32$ \\
\hline F8 & $0.04 \pm 0.07$ & $-0.24 \pm 0.35$ & $0.17 \pm 0.31$ & $0.03 \pm 0.28$ & $0.04 \pm 0.29$ & $-0.01 \pm 0.31$ \\
\hline AF4 & $0.03 \pm 0.07$ & $-0.3 \pm 0.24$ & $0.10 \pm 0.20$ & $-0.13 \pm 0.26$ & $0.1 \pm 0.25$ & $-0.04 \pm 0.28$ \\
\hline LH Hemisphere & $0.04 \pm 0.09$ & $-0.13 \pm 0.17$ & $0.12 \pm 0.13$ & $-0.01 \pm 0.15$ & $0.03 \pm 0.13$ & $0.01 \pm 0.21$ \\
\hline RH Hemisphere & $0.06 \pm 0.06$ & $-0.22 \pm 0.24$ & $0.17 \pm 0.18$ & $-0.05 \pm 0.17$ & $0.04 \pm 0.19$ & $-0.01 \pm 0.21$ \\
\hline Total Brain & $0.06 \pm 0.07$ & $-0.19 \pm 0.21$ & $0.15 \pm 0.15$ & $-0.03 \pm 0.17$ & $0.04 \pm 0.16$ & $0.00 \pm 0.22$ \\
\hline
\end{tabular}

- P8: Increase in $\beta$-high wave (average value $\omega$ : 0.391; average value under ELF-EMF: 1.724, $p=0.049$ ), decrease in Z-Ratio (average value $\omega$ : 0.019; average value under ELF-EMF: $-0.163, \mathrm{p}=0.007)$;

- T8: Increase in $\beta$-low wave (average value $\omega$ : 0.724; average value under ELF-EMF: 2.476, $p=0.043$ ), decrease in Z-Ratio (average value $\omega$ : 0.103; average value under ELF-EMF: $-0.197, \mathrm{p}=0.000$ );

- FC6: Decrease in Z-Ratio (average value $\omega$ : 0.094; average value under ELF-EMF: $-0.267, \mathrm{p}=0.000$ );

- F4: Increase in $\beta$-low wave (average value $\omega$ : 0.788; average value under ELF-EMF: 0.762, $p=0.037$ ), increase in 


\section{$\theta$ BRAIN WAVE POWER BAND}

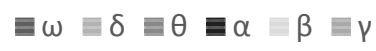
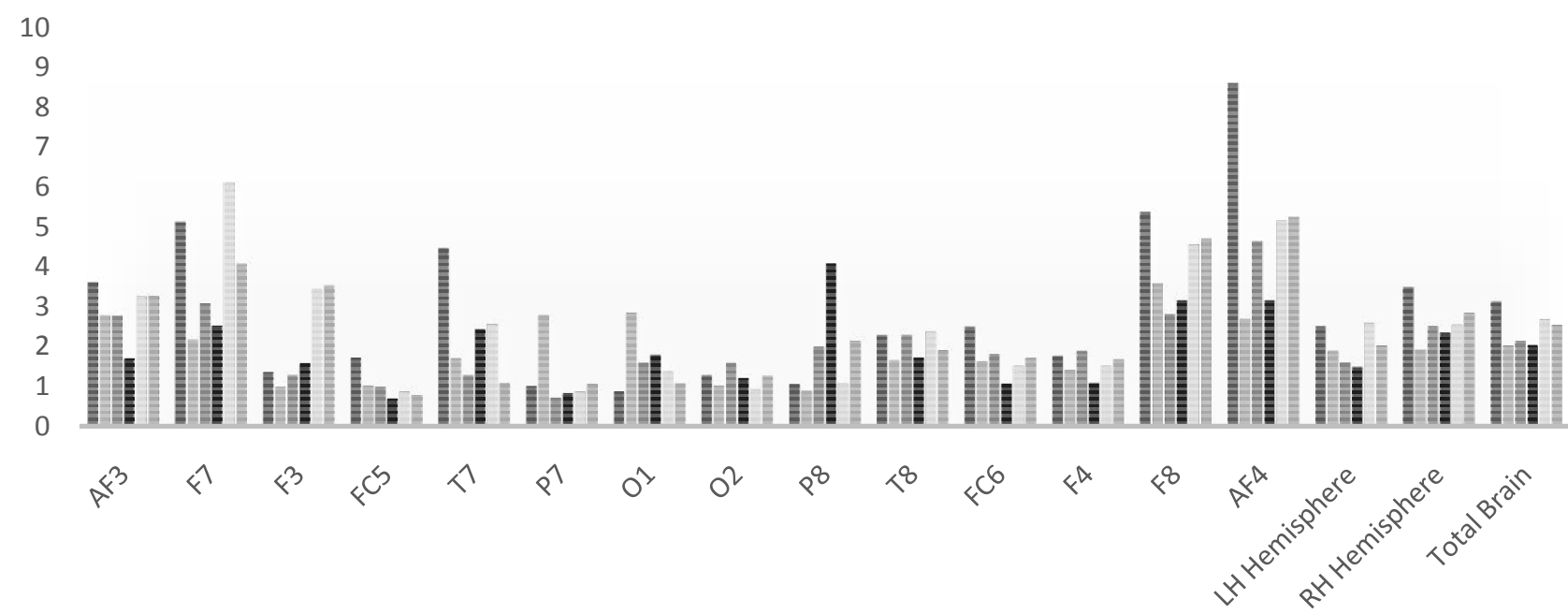

Graph 1: Bar graph of the average values obtained for the 6 different measurements of the $\theta$ brain wave power band (stimulated by ELF-EMF with the exception of $\omega$ ). Each column shows a different endogenous response, with the exception of $\omega$.

\section{a BRAIN WAVE POWER BAND}

$$
\equiv \omega=\delta \equiv \theta \equiv \beta \equiv \gamma
$$

6

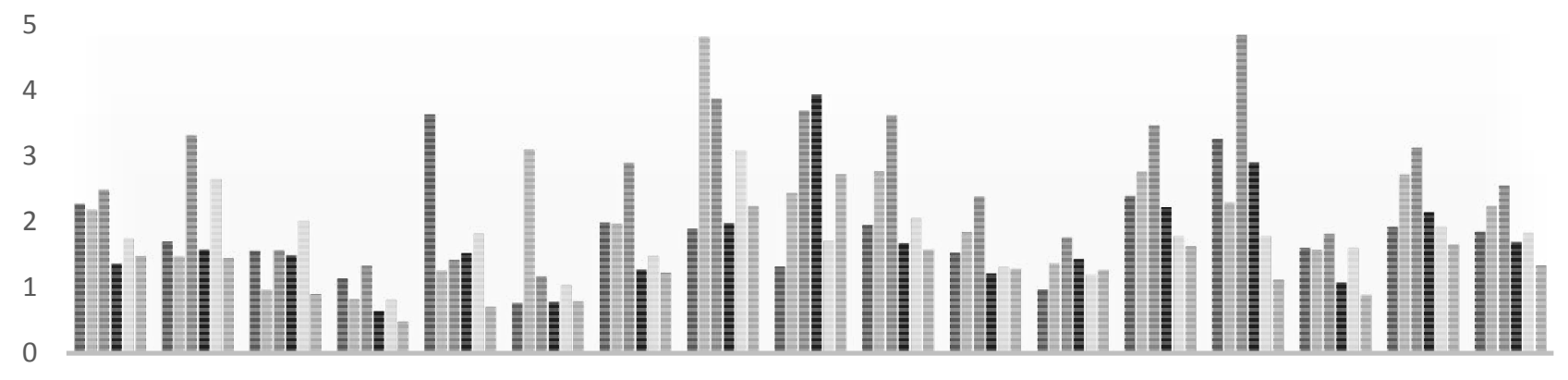

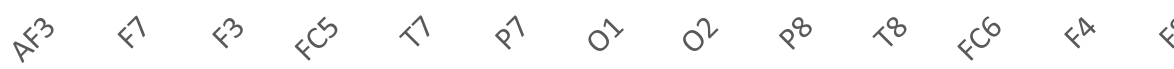

Graph 2: Bar graph of the average values obtained for the 6 different measurements of the $\alpha$ brain wave power band (stimulated by ELF-EMF with the exception of $\omega$ ). Each column shows a different endogenous response, with the exception of $\omega$.

$\beta$-high wave (average value $\omega: 0.471$; average value under ELF-EMF: 0.781, $p=0.022$ ), increase in $\gamma$ wave (average value $\omega$ : 0.153 ; average value under ELF-EMF: $0.445, p=$ 0.000 ), decrease in Z-Ratio (average value $\omega: 0.165$; average value under ELF-EMF: $-0.283, p=0.000$ );

- F8: Decrease in Z-Ratio (average value $\omega: 0.039$; average value under ELF-EMF: -0.242, $p=0.000$ );

- AF4: Increase in $\gamma$ wave (average value $\omega$ : 0.717; average value under ELF-EMF: $1.257, \mathrm{p}=0.025)$, decrease in Z-Ratio (average value $\omega: 0.028$; average value under ELF-EMF: -0.229, $p=0.000)$;

- Average LH: Decrease in Z-Ratio (average value $\omega: 0.042$; average value under ELF-EMF: -0.131, $p=0.000$ );

- Average RH: Increase in $\beta$-low wave (average value $\omega$ : 0.789; average value under ELF-EMF: 2.515, $p=0.05$ ), decrease in Z-Ratio (average value $\omega: 0.063$; average value 


\section{$\beta$-IOW BRAIN WAVE POWER BAND}

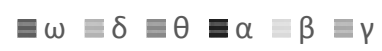

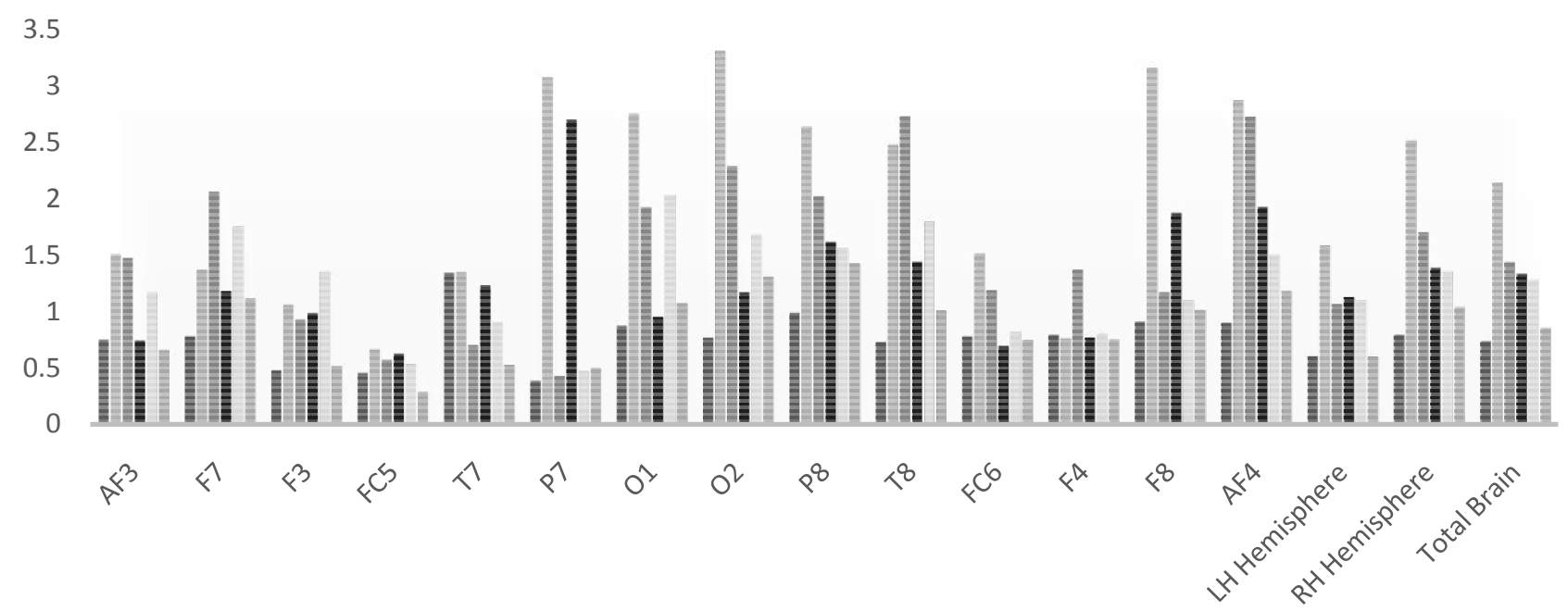

Graph 3: Bar graph of the average values obtained for the 6 different measurements of the $\beta$-low power band (stimulated by ELF-EMF with the exception of $\omega$ ). Each column shows a different endogenous response, with the exception of $\omega$.

\section{$\beta$-high BRAIN WAVE POWER BAND \\ $\equiv \omega \equiv \delta \equiv \theta \equiv \alpha \boxminus \beta \equiv \gamma$}

3

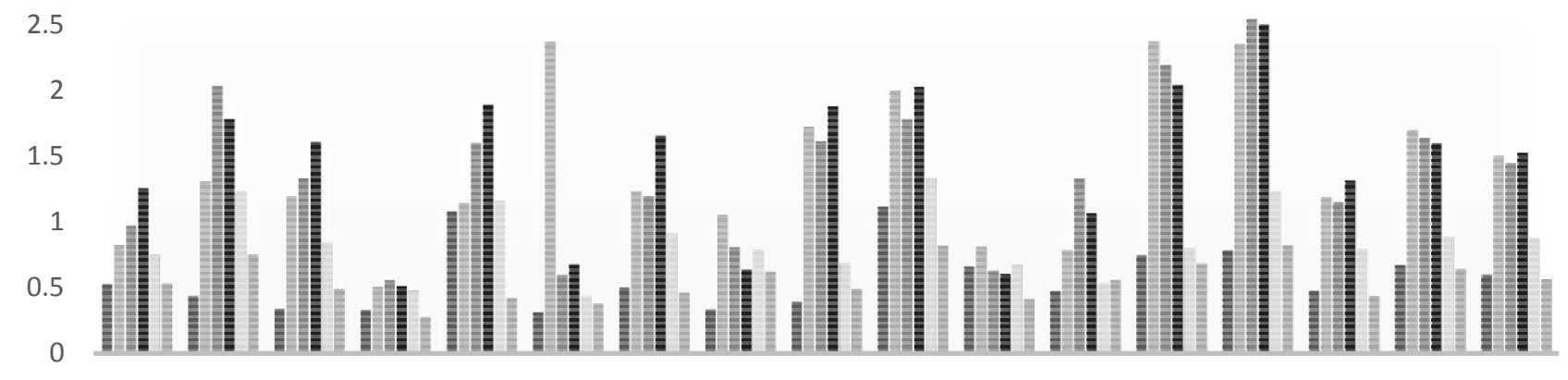

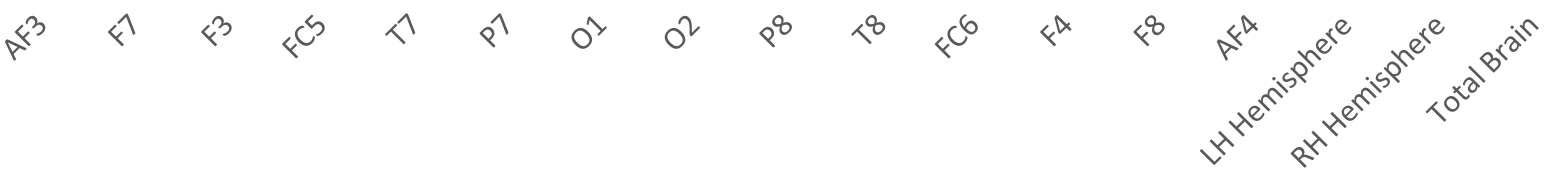

Graph 4: Bar graph of the average values obtained for the 6 different measurements of the $\beta$-high brain wave power band (stimulated by ELF-EMF with the exception of $\omega$ ). Each column shows a different endogenous response, with the exception of $\omega$.

under ELF-EMF: -0.223, $\mathrm{p}=0.000)$;

- Total Brain: Decrease in Z-Ratio (average value $\omega: 0.055$; average value under ELF-EMF: $-0.187, p=0.000$ ).

\section{Effects of stimulation with ELF-EMF $\boldsymbol{\theta}$}

Stimulation with frequencies in the $\theta$ range $(4-6-8 \mathrm{~Hz})$ produced a very different response compared to the stimulation analysed above. In detail:
- AF3: No significant variation in brain waves and Z-Ratio;

- F7: No significant variation in brain waves and Z-Ratio;

- F3: Increase in $\gamma$ wave (average value $\omega$ : 0.229; average value under ELF-EMF: $0.7, \mathrm{p}=0.033$ ), increase in Z-Ratio (average value $\omega$ : 0.038; average value under ELF-EMF: $0.137, \mathrm{p}=0.039)$;

- FC5: No significant variation in brain waves and Z-Ratio; 


\section{Y BRAIN WAVE POWER BAND}

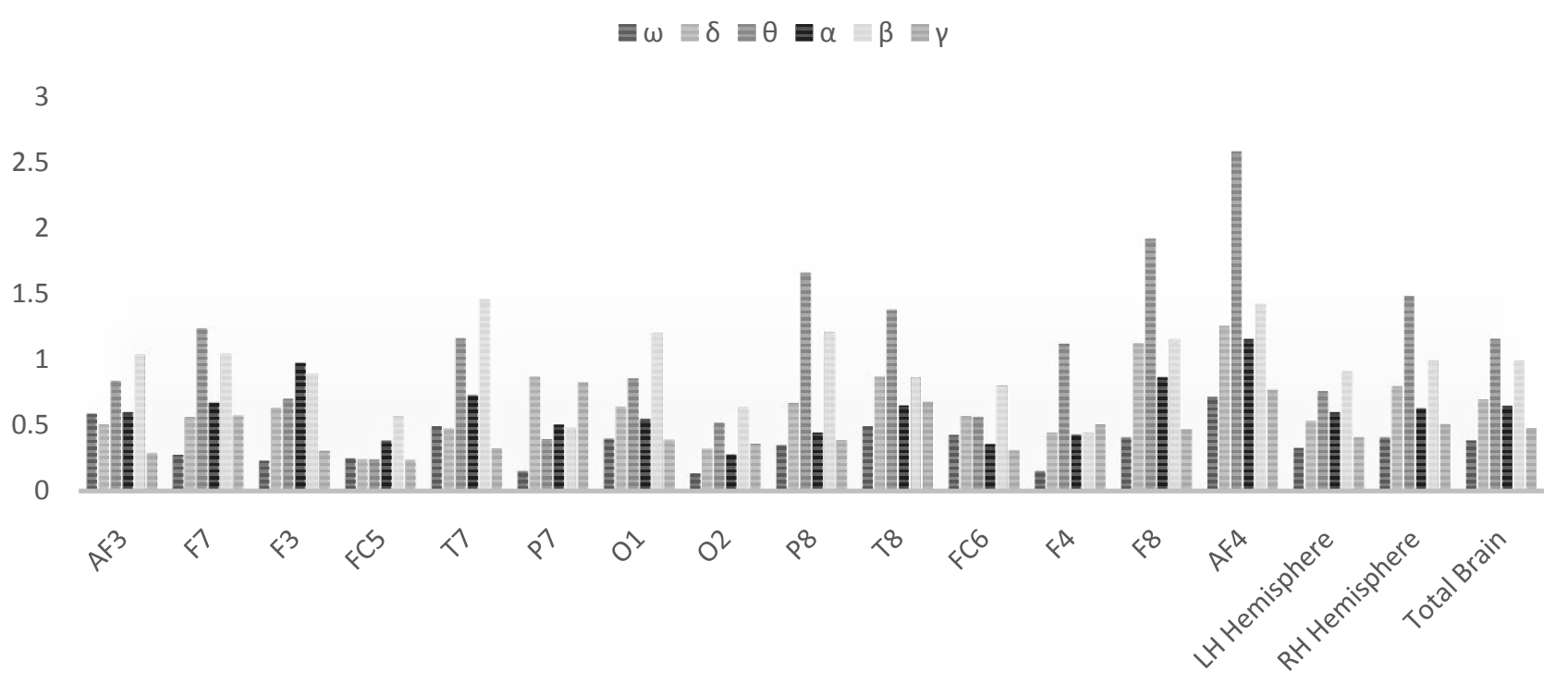

Graph 5: Bar graph of the average values obtained for the 6 different measurements of the $\gamma$ brain wave power band (stimulated by ELF-EMF with the exception of $\omega$ ). Each column shows a different endogenous response, with the exception of $\omega$.

\section{Z-RATIO SCORE \\ $\equiv \omega=\delta \equiv \theta \equiv \beta \equiv \gamma$}

0.3

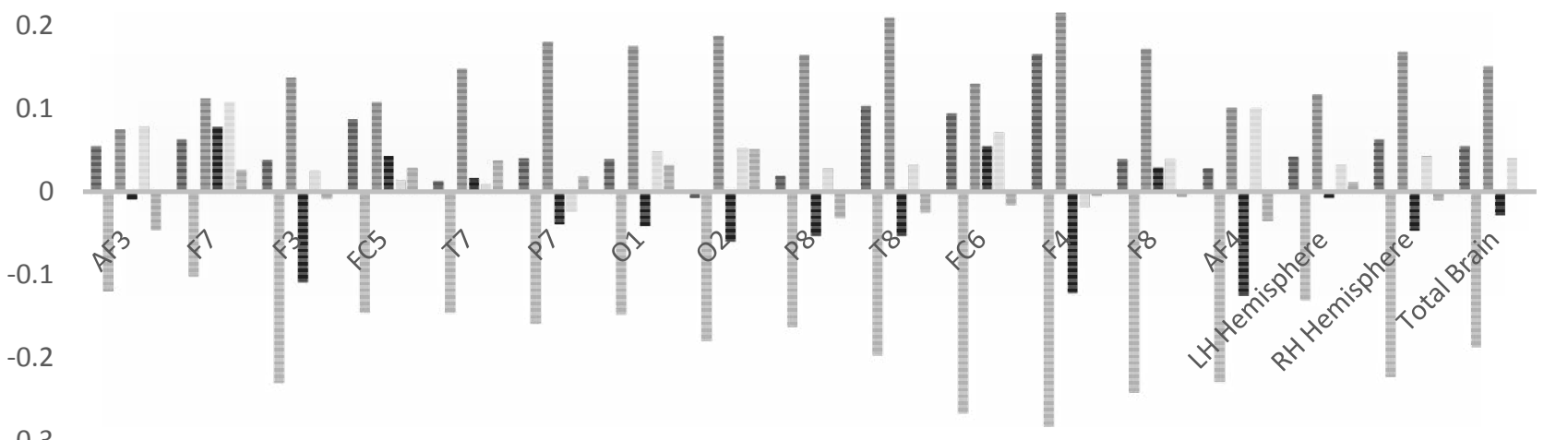

$-0.3$

$-0.4$

Graph 6: Bar graph of the average values obtained for the 6 different measurements of the Z-ratio (stimulated by ELF-EMF with the exception of $\omega$ ). Each column shows a different endogenous response, with the exception of $\omega$.

- T7: Increase in Z-Ratio (average value $\omega: 0.013$; average value under ELF-EMF: 0.148, $\mathrm{p}=0.033$ );

- P7: Increase in $\gamma$ wave (average value $\omega$ : 0.152; average value under ELF-EMF: 0.392, $\mathrm{p}=0.000$ );

- 01: Increase in $\beta$-low wave (average value $\omega$ : 0.872; average value under ELF-EMF: 1.926, $p=0.042$ );

- O2: Increase in $\alpha$ wave (average value $\omega$ : 1.895; average value under ELF-EMF: 3.866, $p=0.016)$, increase in $\beta$-low wave (average value $\omega$ : 0.765 ; average value under ELFEMF: $2.289, p=0.024$ ), increase in $\beta$-high wave (average value $\omega$ : 0.334 ; average value under ELF-EMF: $0.805, p=$ 0.02 ), increase in $\gamma$ wave (average value $\omega: 0.132$; average value under ELF-EMF: 0.518, $p=0.000$ ), increase in Z-Ratio (average value $\omega$ : -0.007 ; average value under ELFEMF: 0.187, $p=0.0137)$; 
- P8: Increase in $\beta$-high wave (average value $\omega$ : 0.391; average value under ELF-EMF: 1.614, $p=0.049)$, increase in Z-Ratio (average value $\omega$ : 0.019; average value under ELFEMF: 0.164, $\mathrm{p}=0.03)$;

- T8: Increase in $\beta$-low wave (average value $\omega$ : 0.724; average value under ELF-EMF: $2.733, p=0.028)$, increase in $\gamma$ wave (average value $\omega: 0.491$; average value under ELFEMF: $1.379, p=0.035$ ), increase in Z-Ratio (average value $\omega: 0.103$; average value under ELF-EMF: 0.209, $p=0.049$ );

- FC6: No significant variation in brain waves and Z-Ratio;

- F4: Increase in $\beta$-low wave (average value $\omega$ : 0.788; average value under ELF-EMF: $1.371, p=0.034)$, increase in $\beta$-high wave (average value $\omega: 0.471$; average value under ELF-EMF: $1.329, p=0.006$ ), increase in $\gamma$ wave (average value $\omega: 0.153$; average value under ELF-EMF: $1.121, \mathrm{p}=$ 0.000 ), increase in Z-Ratio (average value $\omega: 0.165$; average value under ELF-EMF: 0.215, $\mathrm{p}=0.046$ );

- F8: No significant variation in brain waves and Z-Ratio;

- AF4: Increase in $\gamma$ wave (average value $\omega$ : 0.717; average value under ELF-EMF: 2.583, $p=0.042$ ), increase in Z-Ratio (average value $\omega: 0.028$; average value under ELF-EMF: $0.101, p=0.023$ );

- Average LH: No significant variation in brain waves and Z-Ratio;

- Average RH: Increase in $\alpha$ wave (average value $\omega: 1.921$; average value under ELF-EMF: $3.12, p=0.05)$, increase in $\beta$-low wave (average value $\omega$ : 0.789 ; average value under ELF-EMF: $1.702, p=0.017$ ), increase in $\beta$-high wave (average value $\omega$ : 0.672 ; average value under ELF-EMF: 1.639 , $p=0.012$ ), increase in Z-Ratio (average value $\omega: 0.063$; average value under ELF-EMF: 0.168, $p=0.02$ );

- Total Brain: Increase in $\gamma$ wave (average value $\omega: 0.382$ average value under ELF-EMF: 1.158, $p=0.05$ ), increase in Z-Ratio (average value $\omega$ : 0.055 ; average value under ELF-EMF: 0.151, $p=0.023$ ).

\section{Effects of stimulation with ELF-EMF a}

Stimulation with frequencies in the $\alpha$ range $(9-11-13 \mathrm{~Hz}$ ) produced a minor response compared to the two cases described above. In detail:

- AF3: No significant variation in brain waves and Z-Ratio;

- F7: Decrease in $\theta$ wave (average value $\omega$ : 5.117; average value under ELF-EMF: 2.519, $\mathrm{p}=0.045)$;

- F3: Increase in $\gamma$ wave (average value $\omega$ : 0.229; average value under ELF-EMF: 0.974, $p=0.028$ ), decrease in Z-Ratio (average value $\omega$ : 0.038; average value under ELF-EMF: -0.109, $\mathrm{p}=0.03)$;

- FC5: Decrease in $\theta$ wave (average value $\omega: 1.719$; average value under ELF-EMF: 0.695, $\mathrm{p}=0.047$ );

- T7: No significant variation in brain waves and Z-Ratio;

- P7: Increase in $\gamma$ wave (average value $\omega$ : 0.152; average value under ELF-EMF: 0.505, $p=0.011$ ), decrease in Z-Ratio (average value $\omega: 0.04$; average value under ELF-EMF:
-0.039, $p=0.02$ );

- 01: No significant variation in brain waves and Z-Ratio;

- 02: Increase in $\beta$-low wave (average value $\omega$ : 0.765; average value under ELF-EMF: 1.171, $p=0.036)$, increase in $\gamma$ wave (average value $\omega: 0.132$; average value under ELFEMF: 0.276, $p=0.012$ );

- P8: No significant variation in brain waves and Z-Ratio;

- T8: Increase in $\beta$-low wave (average value $\omega$ : 0.724; average value under ELF-EMF: 1.442, $p=0.028$ ), decrease in Z-Ratio (average value $\omega: 0.103$; average value under ELF-EMF: $-0.053, p=0.03$ );

- FC6: Decrease in $\theta$ wave (average value $\omega: 2.498$; average value under ELF-EMF: $1.068, p=0.018$ ), decrease in Z-Ratio (average value $\omega: 0.094$; average value under ELF-EMF: $-0.267, p=0.000)$;

- F4: Relative increase in $\gamma$ wave (average value $\omega: 0.153$; average value under ELF-EMF: 0.429, $p=0.002$ ), decrease in Z-Ratio (average value $\omega$ : 0.165; average value under ELF-EMF: $-0.122, \mathrm{p}=0.000)$;

- F8: No significant variation in brain waves and Z-Ratio;

- AF4: Decrease in Z-Ratio (average value $\omega$ : 0.028; average value under ELF-EMF: $-0.125, p=0.001$ );

- Average LH: Decrease in Z-Ratio (average value $\omega: 0.042$; average value under ELF-EMF: -0.007, $p=0.044$ );

- Average RH: Decrease in Z-Ratio (average value $\omega: 0.063$; average value under ELF-EMF: -0.047, $p=0.000$ );

- Total Brain: Decrease in Z-Ratio (average value $\omega$ : 0.055; average value under ELF-EMF: $-0.028, p=0.004)$.

\section{Effects of stimulation with ELF-EMF $\beta$}

Stimulation with frequencies in the $\boldsymbol{\beta}$ range $(15-22-29 \mathrm{~Hz}$ ) produced a largely coherent response (increased production of the same cerebral frequencies). In detail:

- AF3: No significant variation in brain waves and Z-Ratio;

- F7: Increase in $\beta$-low wave (average value $\omega$ : 0.776; average value under ELF-EMF: $1.75, p=0.05)$, increase in $\beta$-high wave (average value $\omega: 0.433$; average value under ELF-EMF: 1.224, $p=0.026$ ), increase in $\gamma$ wave (average value $\omega: 0.274$; average value under ELF-EMF: $1.042, p=$ 0.0023);

- F3: Increase in $\beta$-high wave (average value $\omega$ : 0.338; average value under ELF-EMF: 0.839, $p=0.005)$, increase in $\gamma$ wave (average value $\omega: 0.229$; average value under ELFEMF: 0.887, $\mathrm{p}=0.003)$;

- FC5: No significant variation in brain waves and Z-Ratio;

- T7: No significant variation in brain waves and Z-Ratio;

- P7: Increase in $\gamma$ wave (average value $\omega: 0.152$; average value under ELF-EMF: 0.497, $p=0.022$ );

- 01: Increase in $\beta$-low wave (average value $\omega: 0.872$; average value under ELF-EMF: 2.032, $p=0.036$ ); 
- O2: Increase in $\beta$-low wave (average value $\omega$ : 0.765 ; average value under ELF-EMF: $1.679, p=0.043)$, increase in $\beta$-high wave (average value $\omega: 0.334$; average value under ELF-EMF: $0.779, p=0.025$ ), increase in $\gamma$ wave (average value $\omega$ : 0.132 ; average value under ELF-EMF: $0.634, p=$ 0.000);

- P8: No significant variation in brain waves and Z-Ratio;

- T8: Increase in $\beta$-low wave (average value $\omega$ : 0.724; average value under ELF-EMF: $1.789, \mathrm{p}=0.049$ );

- FC6: No significant variation in brain waves and Z-Ratio;

- F4: Increase in $\gamma$ wave (average value $\omega$ : 0.153; average value under ELF-EMF: 0.445, $p=0.001$ ), decrease in Z-Ratio (average value $\omega: 0.165$; average value under ELF-EMF: $-0.018, p=0.039)$;

- F8: No significant variation in brain waves and Z-Ratio;

- AF4: Increase in $\gamma$ wave (average value $\omega: 0.717$; average value under ELF-EMF: 1.421, $p=0.043$ );

- Average LH: Increase in $\gamma$ wave (average value $\omega$ : 0.326; average value under ELF-EMF: 0.906, $p=0.048$ );

- Average RH: Increase in $\gamma$ wave (average value $\omega$ : 0.406; average value under ELF-EMF: 0.991, $p=0.044$ );

- Total Brain: Increase in $y$ wave (average value $\omega: 0.382$; average value under ELF-EMF: 0.989, $p=0.044$ ).

\section{Effects of stimulation with ELF-EMF $\gamma$}

Stimulation with frequencies in the $\gamma$ range (31-45-56) $\mathrm{Hz}$ was the range with the least cerebral response in general. In detail:

- AF3: Decrease in Z-Ratio (average value $\omega$ : 0.055; average value under ELF-EMF: -0.046, $p=0.044$ );

- F7: No significant variation in brain waves and Z-Ratio;

- F3: No significant variation in brain waves and Z-Ratio;

- FC5: Decrease in $\alpha$ wave (average value $\omega: 1.136$; average value under ELF-EMF: 0.487, $p=0.043$ );

- T7: No significant variation in brain waves and Z-Ratio;

- P7: No significant variation in brain waves and Z-Ratio;

- 01: No significant variation in brain waves and Z-Ratio;

- 02: Increase in $\gamma$ wave (average value $\omega: 0.132$; average value under ELF-EMF: 0.36, $\mathrm{p}=0.003$ );

- P8: No significant variation in brain waves and Z-Ratio;

- T8: No significant variation in brain waves and Z-Ratio;

- FC6: No significant variation in brain waves and Z-Ratio;

- F4: Increase in $\gamma$ wave (average value $\omega$ : 0.153; average value under ELF-EMF: 0.505, $p=0.000$ ), decrease in Z-Ratio (average value $\omega: 0.165$; average value under ELF-EMF: $-0.005, p=0.019)$;

- F8: No significant variation in brain waves and Z-Ratio;

- AF4: Decrease in a wave (average value $\omega$ : 3.255; average value under ELF-EMF: 1.121, $\mathrm{p}=0.034$ );

- Average LH: No significant variation in brain waves and Z-Ratio;

- Average RH: Decrease in Z-Ratio (average value $\omega: 0.063$; average value under ELF-EMF: -0.01, $p=0.032$ );

- Total Brain: No significant variation in brain waves and Z-Ratio.

\section{General Discussion}

In a world in which technologies based on EMF are increasingly fundamental in numerous human activities, understanding how, and to what extent our CNS is sensitive to this exposure has become essential. Within a range of very low frequencies and intensities, those of the ELF-EMF, significant biological responses have been observed, like for example a reduction in oxidative stress [16-21], myeloprotection [18], stimulation of tissue regeneration [22,23], diverse effects on the heart rate variability index or HRV [11], and in the psychiatric sphere [10]. Some experience is also starting to accumulate on the direct effects of ELFEMF on the CNS, through observation of variations in cerebral electrical activity [5-9].

The response of the CNS to ELF-EMF has also been assessed from a biochemical perspective in literature. Daily non-focused stimulation for 1 hour over 14 days generated an increase in dopamine and serotonin turnover in treated mice [24]. Longer daily stimulations of mice led to increased production of $\beta$-endorphin and substance $P$ [25]. Concrete evidence of biological sensitivity to ELF-EMF, and the effects recorded in a previous study on the ANS through analysis of HRV [11], induced the authors to investigate the effects of non-focused treatment on cerebral electrical activity.

The study was conducted on all the subjects after their working hours and consequently in a condition of relatively high fatigue and stress. The measurements were taken from January 2018 up to January 2019. The experimental set-up involved the use of the same SEQEX ${ }^{\circledR}$ device with the transmitting mat on top of a wooden massage couch in order to avoid the generation of spurious currents, and positioned so that the subjects could not see whether or not the device was effectively emitting ELF-EMF. Given that the treatment does not produce any sound or bodily sensation, the subjects were unable to know whether treatment was being administered or only simulated. In order to avoid a summation effect from the various treatments, after each electromagnetic set-up the subjects were asked to stand up away from the mat for 3 minutes.

The response recorded in the EEG was different for each electromagnetic set-up. Firstly, it is important to note that there was higher total stimulation at the extremely low frequencies (1-3 Hz), with a response recorded in all the derivations studied, as well as in the sum of the derivations per hemisphere. In contrast, the higher frequencies $(31-56 \mathrm{~Hz})$ stimulated a lower cerebral electrical response. It could be hypothesised that, given the considerable difference in amplitude of endogenous delta waves, 
the whole-brain response could be caused by a cascade mechanism that triggers various Hyper-Neurons due to the background amplitude of the delta frequencies [26].

Furthermore, endogenous delta waves are typical of sleep and rest, a physiological activity that naturally restores cerebral function, as deduced from studies on the restorative effects of sleep and the harmful effects of sleep abstinence [27].

In general, the right hemisphere derivations exhibited greater responsiveness to stimulation. It could be hypothesised that rhythmic stimuli, like musical stimuli, may activate the right hemisphere cortex more strongly [28].

The data are reported in detail below, associating the derivations studied with the correlated cerebral functions according to literature [29-36].

Specifically, the administration of ELF-EMF in the 1-3 Hz range led to a general increase in $\beta$ activity in the motor area $\left(\mathrm{F} 3, \mathrm{p}_{\beta \text {-low }}<0.05\right.$ and $\left.\mathrm{p}_{\beta \text {-high }}<0.05\right)$, but mostly in the right hemisphere visual area $\left(02, p_{\beta \text {-low }}=0.05\right.$ and $\left.p_{\beta \text {-high }}<0.01\right)$, sensory integration area $\left(P 8, \mathrm{p}_{\beta \text {-high }}<0.05\right)$, auditory area (T8, $\left.\mathrm{p}_{\beta \text {-low }}<0.05\right)$, and motor area $\left(F 4, \mathrm{p}_{\beta \text {-low }}<0.05\right.$ e $\left.\mathrm{p}_{\beta \text {-high }}<0.05\right)$.

In the left $\left(F 3, p_{\gamma}<0.05\right)$ and right $\left(F 4, p_{\gamma}<0.05\right)$ frontal areas a significant increase in $\gamma$ activity was recorded. This cortical hyper-activation was also recorded in the left sensory integration area $(\mathrm{P} 7, \mathrm{p} \gamma<0.05)$ as well as the visual area $(02$, $\left.\mathrm{p}_{\gamma}<0.05\right)$ and right frontal associative zone (AF4, $\left.\mathrm{p}_{\gamma}<0.05\right)$. $A$ significant decrease in Z-ratio was recorded in all the derivations $(0.000 \leq p<0.02)$. This observation offers interesting interpretative speculations: while on one hand cerebral activity obviously increased in some derivations, on the other hand it is possible that in the others it was the decrease in low frequency components that produced the Z-ratio result. Thus in general the response of the CNS to the $1-3 \mathrm{~Hz}$ range is adaptive in nature: It thus does not respond by increasing the analogous frequencies (there is no frequency following response or FFT mechanism), but instead globally amplifies compensatory electrical activity in many areas.

In contrast, the administration of ELF-EMF within the $\boldsymbol{\theta}$ range of 4-8 $\mathrm{Hz}$ generated increased $\beta$ activity in the $\mathrm{RH}$ hemisphere, and in particular a considerable increase in Z-ratio. Under stimulation in the $4-8 \mathrm{~Hz}$ range, $\beta$ activity increased bilaterally in the occipital area $\left(01, p_{\beta \text {-low }}<0.05\right.$ and $02, \mathrm{p}_{\beta \text {-low }}<0.05$ e $\left.\mathrm{p}_{\beta \text {-high }}<0.05\right)$, and on the right in the sensory integration area ( $\left.P 8, p_{\beta \text {-high }}<0.05\right)$, auditory area $\left(T 8, \mathrm{p}_{\beta \text {-low }}<0.05\right)$, and motor area $\left(\mathrm{F} 4, \mathrm{p}_{\beta \text {-low }}<0.05\right.$ and $\mathrm{p}_{\beta \text {-high }}$ $<0.01)$.

The central frontal $\theta$ waves in human EEG are often considered to be of hippocampal origin. In mammals the most obvious characteristic of the hippocampus is the capacity to generate theta waves [37]. This rhythm can be measured globally with macro-electrodes in all parts of the hippocampus and in the majority of interconnected anatomic structures [37]. This observed extracellular phenomenon reflects the cooperative behaviour of large numbers of pyramidal cells in the hippocampus [37]. Hippocampal pyramidal cells activate periodically and in synchrony to produce theta oscillations. The frequency of these oscillations vary across quite a wide range from 4 to $10 \mathrm{~Hz}$. This class of oscillations are usually grouped together under the term "hippocampal theta waves". These waves appear to play a role in the neural codification of place [38]. Orientation in a location activates the "place cells" of the hippocampus. This information can considerably improve the precision of reconstruction of the position of an animal in space, also coded with visual stimuli, explaining the activation of $\beta$ waves in visual areas like 02 and 01 . $\theta$ activation plays an important role in sensory and motor integration during exploratory research and during behaviours motivated by visual objectives [39]. It is thus the hypothesis of the authors that stimulation within the $\theta$ range, generating an overall response, might produce increased hippocampal involvement with consequent positive effects on the functions described immediately above and on adaptive intelligence.

$\gamma$ activation by ELF-EMF in the $4-8 \mathrm{~Hz}$ range again affected the right hemisphere more than the left. There was a bilateral response of the motor areas (F3 $p_{y}<0.05$ and F4 $\left.p_{v}<0.01\right)$, and ipsilateral left response of the sensory integration area $\left(P 7, p_{\gamma}<0.01\right)$, while on the right there was a response in the visual area $\left(02, p_{\gamma}<0.01\right)$, auditory area $\left(T 8, p_{\gamma}<0.05\right)$, and frontal associative zone (AF4, $\left.p_{\gamma}<0.05\right)$. As regards the right visual area, also of note was an activation within the $\alpha$ range $\left(02, p_{\alpha}<0.05\right)$. In contrast with the results from the previous stimulation, the Z-ratio was not significantly modified in any of the derivations studied, and above all the statistical significance was inverse with the Z-ratio value increased, thus indicating a relative increase in low waves $(\delta)$. The response was thus different from the one analysed previously: It is probable that, in this case, instead of an adaptive response there was a coherent response (low frequency stimulation producing a low frequency response).

The administration of ELF-EMF in the $\alpha$ range $(9-13 \mathrm{~Hz})$, generated a lower response compared to the 2 previous stimulations. However, within the $\beta$ wave stimulation range, a significant increase was recorded only on the right, and specifically $\beta$-low in the visual area $\left(02, \mathrm{p}_{\beta \text {-low }}<0.05\right)$ and auditory area $\left(T 8, p_{\beta-\text { low }}<0.05\right)$. Once again it is reasonable to assume a natural adaptive response of the nervous system to waves in the alpha range, as inducers of a "relaxed alert" state. Other methods apply a focus algorithm that indicates intensity of mental "focus" or "attention" [40].

In the frequencies between 13 and $30 \mathrm{~Hz}$ beta waves are more frequently observed in the frontal or central areas than in the rear areas of the cortex [40]. At least two distinct beta waves can be separated: Beta waves with maximum electroencephalograph spectra (EEG) above the sensory and motor strip (Rolandic beta rhythms), and beta waves located more frontally (frontal beta waves) [40]. These waves are expressed as peaks on the individual spectrums. However, plenty of healthy subjects exhibit distinct peaks on the spectra, and consequently wide average spectra with eyes both open and closed do not demonstrate clear maximums in the beta 
frequency band [40]. The amplitude of the beta waves measured with reference to the associated ears is less than 20 $\mu \mathrm{V}$. A $\beta$-low response in the visual area $\left(02, \mathrm{p}_{\beta \text {-low }}<0.05\right)$ and auditory area $\left(T 8, \mathrm{p}_{\beta \text {-low }}<0.05\right)$ is thus probably artificial and induced by EM stimulation in the $9-13 \mathrm{~Hz}$ range. The effect induced could thus be attention without focus or listening.

An increase in $\gamma$ activity was also observed, again bilaterally in the motor areas ( $F 3 p_{\gamma}<0.05$ and F4 $\left.p_{\gamma}<0.01\right)$, left sensory integration area $\left(P 7, p_{\gamma}<0.05\right)$, and right visual area $(02$, $\left.\mathrm{p}_{\gamma}<0.01\right)$. A drop was noted in the power of the $\theta$ band bilaterally in the mnemonic/motor area (FC5 $p_{\theta}<0.05$ and FC6 $\mathrm{p} \theta<0.05)$, and left verbal and associative area (F7, $\left.\mathrm{p}_{\theta}<0.05\right)$. The Z-ratio responded in a similar way as when stimulated with 1-3 Hz, with increased activity in the higher cerebral frequencies. However, the area involved was less extensive and mostly in the right hemisphere. The Z-ratio value fell bilaterally in the motor areas $\left(F 3 p_{z}<0.05\right.$ and $\left.F 4 p_{z}<0.01\right)$, on the left in the sensory integration area $\left(P 7, p_{z}<0.05\right)$, on the right in the auditory area $\left(T 8, p_{z}<0.05\right)$, mnemonic/motor area (FC6 $\left.\mathrm{p}_{\mathrm{z}}<0.01\right)$, and frontal associative area (AF4, $\left.\mathrm{p}_{\mathrm{z}}<0.01\right)$. Consequently, administration in the $\alpha$ range $(9-13 \mathrm{~Hz})$ can be assessed as a possible inducer of sensory alertness.

Administration of ELF-EMF in the $15-29 \mathrm{~Hz}$ range generated a substantially coherent response (i.e. production of cerebral waves with similar frequencies to those administered). There was an increase in the $\beta$ band, this time mainly on the left. There were some very interesting divergences from expected reactions, with a suppression or desynchronization of occipital alpha waves when frontal $\beta$ waves were activated. In detail, on the left there was a significant increase in $\beta$ in the verbal and associative area $\left(F 7, p_{\beta \text {-low }}<0.05\right.$ and $\left.p_{\beta \text {-high }}<0.05\right)$, motor area $\left(F 3 p_{\gamma}<\right.$ $0.01)$, and visual area $\left(01, \mathrm{p}_{\beta-\text { low }}<0.05\right)$; the motor component $\left(\mathbf{F} 3 \mathbf{p}_{\gamma}<\mathbf{0 . 0 1}\right)$ mirrored the typically frontal functions (those exclusively motoric would vary readings for $\mathrm{C} 3$, and the Rolandic $\beta$ rhythm is modulated during various motor and cognitive tasks). Conversely, beta waves are normally expressed in spectrograms of low amplitude and irregular pattern. Beta waves almost never exceed $20 \mu \mathrm{V}$. In F3, Fz, and $F 4$ the EEG spectra generally show maximum response around $19 \mathrm{~Hz}$.

The right side principally saw increased activity in the visual area $\left(02, \mathrm{p}_{\beta \text {-low }}<0.05\right.$ and $\left.\mathrm{p}_{\beta \text {-high }}<0.05\right)$ and auditory area $\left(T 8, p_{\beta-\text { low }}<0.05\right)$. The response in the $\gamma$ band was wider, bilaterally involving the motor areas $\left(F 3 p_{\gamma}<0.01\right.$ and F4 $\left.p_{\gamma}<0.01\right)$, on the left the verbal and associative area $\left(F 7, p_{\gamma}<0.01\right)$ and sensory integration area $\left(P 7, p_{\gamma}<\right.$ $0.05)$, while on the right there was a response in the visual area $\left(02, p_{\gamma}<0.01\right)$ and frontal associative area (AF4, $p_{y}$ $<0.05)$. The Z-ratio was significantly reduced, with consequent generalized increase in frequencies, only in the right motor area $\left(F 4 \mathrm{p}_{\mathrm{z}}<0.05\right)$.

ELF-EMF stimulation in the $31-56 \mathrm{~Hz}$ range generated a low adaptive response, largely coherent in nature, as in the previous case. The only areas of significant response (i.e. increased amplitude) were recorded on the right for $\gamma$ waves in the visual area $\left(02, \mathrm{p}_{\gamma}<0.01\right)$ and motor area (F4 $\left.\mathrm{p}_{\gamma}<0.01\right)$. Also of note was a fall in the $\alpha$ band in the right mnemonic/ motor area (FC5 $\left.p_{\alpha}<0.05\right)$ and right frontal associative area (AF4 $p_{\alpha}<0.05$ ). It is possible to hypothesise a bland effect of these ranges on the residual and spurious hippocampal response with registration in the cortical area. A decrease in the Z-ratio, indicating an entirely coherent response, was recorded in the left frontal associative area (AF4 $\left.p_{z}<0.05\right)$ and right motor area $\left(F 4 \mathrm{p}_{\mathrm{z}}<0.05\right)$.

The responses to the various non-focused electromagnetic stimuli offer interesting pointers for reflection, which can be summarized as follows:

1. This study and all similar literature already published [6-9], confirms the high sensitivity of the human nervous system to electromagnetic fields. EMF are generated endogenously or perceived on the entire body from natural and artificial irradiation, received in a "non-focused" manner. This poses important questions in regards to the health implications of electromagnetic pollution or disturbances that characterizes modern life. A study in 2001 [41] highlighted how the incidence of some of the most common ailments (like headache, fatigue, insomnia, nervousness, etc.) is higher among populations living in proximity to cellular phone relay stations, and decreases moving away from sources of irradiation. The ever clearer emerging evidence of the interaction between electromagnetic fields and biology, makes it necessary to reassess the incidence of disorders due to civil exposure to electromagnetic frequencies and the impact that these might have on public health. Electromagnetic pollution should be considered on a par with chemical pollution as a genuine pathogenic factor to be investigated and classified.

2. The evidence of effects both on the CNS, as demonstrated in the present study, and on the ANS [11], offers interesting cues for understanding results obtained from other experiences with non-focused ELF-EMF, like for example in psychiatry [10]. It is probable that an improved balance between the two ANS components, combined with an increase of cerebral $\beta$ waves, underlies the therapeutic response in this class of subjects. To date this is still only a logically based conjecture, which the authors believe deserves further investigation.

3. The sensitivity exhibited by the CNS and ANS [11] oblige a review of the manner in which ELF-EMF are used today for therapeutic purposes. It is no longer possible to adopt a reductionist approach to this type of therapy. The authors believe that it would be opportune to diverge from the idea of treating only pathological aspects (i.e. bone fractures, osteoarticular pain, etc.), since whenever a subject is administered non-focused ELF-EMF there is a response not only in the part of the body under treatment, but of the entire nervous system. This response becomes an integral part of the therapy and always needs to be taken into consideration.

4. The CNS is seen to respond variably to different elec- 
tromagnetic stimuli. Surprisingly, the most important response was to the lowest field frequencies, which induced an adaptive response in the CNS, with an increase in the power of the $\beta$ band as well as a balanced decrease in Z-ratio. As frequency increased the cortical response changed from adaptive to coherent, as if the CNS and ELFEMF had started to synchronize. The response to the 4-6$8 \mathrm{~Hz}$ frequencies was interesting, with activation mainly on the right, but above all with an increase (the only case recorded during the study) of the Z-ratio in various brain derivations. Lack of power in the $\delta$ band due to limits in the software used for EEG analysis means that it cannot be confirmed with certainty that the Z-ratio figure is due to a significant increase in that specific band, even though this does seem to be the most likely hypothesis.

5. A difference of response between the two hemispheres appears very clear. Based on the observations of the present study, the right hemisphere appears more susceptible to electromagnetic stimulation than the left hemisphere. There is also a substantial difference in response between occipital and frontal regions, the former being much more susceptible than the latter.

This research, which completes the study on the ANS, represents a first step towards better understanding variability of response to different frequency ranges of ELF$E M F$, with the aim of achieving greater knowledge of the systemic effects of these increasingly widespread therapies.

It could be hypothesised that, if the present results are confirmed in further studies, the interaction between ELFEMF and cerebral electrical state could offer major opportunities against a whole series of disturbances in which the lack of one or more waves represents the main sign of disease (for example children with ADHD [42], patients with insomnia, fibromyalgia, etc.). Considering the high correlation between cerebral waves and psychiatric disorders like depression [43], anxiety [43], or post-traumatic stress syndrome [44], and given the evidence (to be extended and confirmed) of this type of approach in psychiatry [10], it is possible to propose integrated use in a range of situations in which conventional approaches do not always achieve satisfactory results.

\section{Conclusions}

The CNS is seen to be variably sensitive to ELF-EMF, according to the frequencies used, even under brief stimulations. It is thus possible that some of the therapeutic effects of non-focused ELF-EMF experienced by patients should be attributed to modifications in cerebral electrical behaviour, with both therapeutic and pathophysiological implications (i.e. electromagnetic pollution) that should be taken seriously.

\section{References}

1. Vecchia P (2007) Exposure of humans to electromagnetic fields. Standards and regulations. Ann Ist Super Sanita 43: 260-267.

2. Gorelick DA, Zangen A, George MS (2014) Transcranial magnetic stimulation in the treatment of substance addiction. Ann N Y Acad Sci 1327: 79-93.
3. Nadine Dougall, Nicola Maayan, Karla Soares-Weiser, et al. (2015) Transcranial magnetic stimulation for schizophrenia. Schizophrenia Bulletin 41: 1220-1222.

4. Narayana S, Papanicolaou AC, McGregor A, et al. (2015) Clinical applications of transcranial magnetic stimulation in pediatric neurology. J Child Neurol 30: 1111-1124.

5. Farzan F, Vernet M, Shafi MM, et al. (2016) Characterizing and modulating brain circuitry through transcranial magnetic stimulation combined with electroencephalography. Front Neural Circuits 10: 73.

6. Cretkovic D, Jovanov E, Cosic I (2006) Alterations in human EEG activity caused by extremely low frequency electromagnetic fields. Conf Proc IEEE Eng Med Biol Soc 1: 3206-3209.

7. Hausser K, Tellschaft D, Thoss F (1997) Influence of an alternating $3 \mathrm{~Hz}$ magnetic field with an induction of $0.1 \mathrm{mT}$ on chosen parameters of the human occipital EEG. Neurosci Lett 239: 57-60.

8. Shafiei SA, Firoozabadi SM, Rasoulzadeh TK (2012) Study of the frequency parameters of EEG influenced by zone-dependent local ELF-MF exposure on the human head. Electromagn Biol Med 31: $112-121$.

9. Shafiei SA, Firoozabadi SM, Tabatabaie KR, et al. (2014) Investigation of EEG changes during exposure to extremely low-frequency magnetic field to conduct brain signals. Neurol Sci 35: 1715-1721.

10. Betti, Marco PCP, Marco Saettoni, et al. (2019) Ion cyclotron resonance: Results and prospects for psychiatry. J Psychiatry Treat res 1: $16-24$.

11. Greco A, Destefani. A (2018) Effects of non-focused Elf-Emf treatment on Hrv: Preliminary study. International Journal of Depression and Anxiety 1.

12. Pravdich NVV (1913) Ein Versuch der Registrierung der elektrischen Gehirnerscheinungen (In German). Zbl Physiol 27: 951960.

13. Badcock NA, Mousikou P, Mahajan Y, et al. (2013) Validation of the emotiv $\mathrm{EPOC}\left({ }^{\circledR}\right) \mathrm{EEG}$ gaming system for measuring research quality auditory ERPs. PeerJ 1: e38.

14. Badcock NA, Preece KA, de Wit B, et al. (2015) Validation of the emotiv EPOC EEG system for research quality auditory event-related potentials in children. PeerJ 3: e907.

15. Albertario CL, Zendell SM, Hertz G, et al. (1995) Comparison of a frequency-based analysis of electroencephalograms (Z-ratio) and visual scoring on the multiple sleep latency test. Sleep 18: 836-843.

16. Li JP, Chen S, Peng H, et al. (2014) Pulsed electromagnetic fields protect the balance between adipogenesis and osteogenesis on steroid-induced osteonecrosis of femoral head at the pre-collapse stage in rats. Bioelectromagnetics 35: 170-180.

17. Morabito C, Rovetta F, Bizzarri M, et al. (2010) Modulation of redox status and calcium handling by extremely low frequency electromagnetic fields in $\mathrm{C} 2 \mathrm{C} 12$ muscle cells: A real-time, single-cell approach. Free Radic Biol Med. 48: 579-589.

18. Rossi E, Corsetti MT, Sukkar S, et al. (2007) Extremely low frequency electromagnetic fields prevent chemotherapy induced myelotoxicity. Electromagn Biol Med 26: 277-281.

19. Raggi F, Vallesi G, Rufini S, et al. (2008) ELF magnetic therapy and oxidative balance. Electromagn Biol Med 27: 325-339.

20. Vallesi G, Raggi F, Rufini S, et al. (2007) Effects of cyclotronic ion 
resonance on human metabolic processes: A clinical trial and one case report. Electromagn Biol Med 26: 283-288.

21. Rauš Balind S, Selaković V, Radenović L, et al. (2014) Extremely low frequency magnetic field $(50 \mathrm{~Hz}, 0.5 \mathrm{mT})$ reduces oxidative stress in the brain of gerbils submitted to global cerebral ischemia. PLoS One 9: e88921.

22. Das S, Kumar S, Jain S, et al. (2012) Exposure to ELF- magnetic field promotes restoration of sensori-motor functions in adult rats with hemisection of thoracic spinal cord. Electromagn Biol Med 31: 180-194.

23. Zhong C, Zhao TF, Xu ZJ, et al. (2012) Effects of electromagnetic fields on bone regeneration in experimental and clinical studies: A review of the literature. Chin Med J (Engl) 125: 367-372.

24. Sieroń A, Labus $\measuredangle$, Nowak P, et al. (2004) Alternating extremely low frequency magnetic field increases turnover of dopamine and serotonin in rat frontal cortex. Bioelectromagnetics 25: 426430.

25. Bao X, Shi Y, Huo X, et al. (2006) A possible involvement of b-endorphin, substance $p$, and serotonin in rat analgesia induced by extremely low frequency magnetic field. Bioelectromagnetics 27: 467-472.

26. Başar E, Düzgün A (2016) The CLAIR model: Extension of brodmann's areas based on brain oscillations and connectivity. Int J Psychophysiol 103: 185-198.

27. Alhola P, Polo-Kantola P (2007) Sleep deprivation: Impact on cognitive performance. Neuropsychiatr Dis Treat 3: 553-567.

28. M Tervaniemia, T Ilvonen, $\mathrm{K}$ karma, et al. (1997) The musical brain: Brain waves reveal the neurophysiological basis of musicality in human subjects. Neuroscience Letters 226: 1-4.

29. Cook IA, O'Hara R, Uijtdehaage SH, et al. (1998) Assessing the accuracy of topographic EEG mapping for determining local brain function. Electroencephalogr Clin Neurophysiol 107: 408-414.

30. Vidal F, Burle B, Spieser L, et al. (2015) Linking EEG signals, brain functions and mental operations: Advantages of the Laplacian transformation. Int J Psychophysiol 97: 221-232.

31. Duffy FH (1989) Clinical value of topographic mapping and quantified neurophysiology. Arch Neurol 46: 1133-1134.

32. Thatcher $R$ (2010) Validity and reliability of quantitative electroencephalography. Journal of Neurotherapy 14: 122-152.
33. Thatcher R, Lubar J (2008) History of the scientific standards of QEEG normative databases. In Thomas Budzinsky, H Budzinski, J Evans, et al. Introduction to QEEG and neurofeedback: Advanced theory and applications, ( $2^{\text {nd }}$ edn), Academic Press, San Diego, 29-62.

34. Thatcher R, RA Walker, CJ Biver, et al. (2003) Sensitivity and specificity of an EEG normative data base: Validation and clinical correlation. J Neurotherapy 7: 87-121.

35. Tramontano G (2006) QEEG testing can discern reason for cognitive disorder: Digital EEG recordings of brainwaves can determine TBI etiology. Connecticut Lawyer, 14-16.

36. Nuwer M (1997) Assessment of digital EEG, quantitative EEG, and EEG brain mapping: Report of the american academy of neurology and the american clinical neurophysiology society. Neurology 49: 277-292.

37. Juri DK (2009) Quantitative EEG, event-related potentials and neurotherapy.

38. O'Keefe J, Recce ML (1993) Phase relationship between hippocampal place units and the EEG theta rhythm. Hippocampus 3: 317-330.

39. Bland BH, Oddie SD (2001) Theta band oscillation and synchrony in the hippocampal formation and associated structures: The case for its role in sensorimotor integration. Behav Brain Res 127: 119-136.

40. Felzer T, Freisleben B (2003) Analyzing EEG signals using the probability estimating guarded neural classifier. IEEE Trans Neural Syst Rehabil Eng 11: 361-371.

41. Santini R, Santini P, Seigne M, et al. (2001) Symptômes exprimés par des riverains de stations relais de téléphonie mobile. Presse Med 30: 1594.

42. Lenartowicz A, Loo SK (2014) Use of EEG to diagnose ADHD. Curr Psychiatry Rep 16: 498.

43. Al Zoubi O, Ahmad M, Aki T, et al. (2019) EEG microstates temporal dynamics differentiate individuals with mood and anxiety disorders from healthy subjects. Front Hum Neurosci 13: 56.

44. Mo HM, Ryan AO, Kristianna BW, et al. (2019) Strong correlation of novel sleep electroencephalography coherence markers with diagnosis and severity of posttraumatic stress disorder. Sci Rep.

DOI: $10.36959 / 817 / 524$

Copyright: (C) 2019 Greco A, et al. This is an open-access article distributed under the terms of the Creative Commons Attribution License, which permits unrestricted use, distribution, and reproduction in any medium, provided the original author and source are credited. 